Cahiers de recherches médiévales

Journal of medieval studies

$18 \mid 2009$

Le système d'enseignement occidental $\left(\mathrm{Xl}^{\mathrm{e}}-\left.\mathrm{XV}\right|^{\mathrm{e}}\right.$ siècle)

\title{
Entre Histoire et mythe
}

Quand il faut être un héros pour être hors-la-loi

\section{Myriam White-Le Goff}

\section{(2) OpenEdition \\ Journals}

Édition électronique

URL : https://journals.openedition.org/crm/11714

DOI : $10.4000 / \mathrm{crm} .11714$

ISSN : $1955-2424$

Éditeur

Honoré Champion

Édition imprimée

Date de publication : 20 novembre 2009

Pagination : 293-305

ISSN : $1272-9752$

Référence électronique

Myriam White-Le Goff, «Entre Histoire et mythe », Cahiers de recherches médiévales [En ligne], 18|

2009, mis en ligne le 15 décembre 2012, consulté le 15 décembre 2022. URL : http://

journals.openedition.org/crm/11714; DOI : https://doi.org/10.4000/crm.11714 


\title{
酷RM
}

\section{Entre Histoire et mythe: quand il faut être un héros pour être hors-la-loi}

\begin{abstract}
The article concerns Fouke Fitz-Warin, the outlaw stereotype often associated with Robin Hood. It questions the meaning of law and the limit of its legitimacy as well as the way in which an outlaw can paradoxically incarnate honesty and justice. This reversal is only possible, literarily speaking, because the character is not a simple revolting baron but rather someone rising to the realms of the mythical hero even if, in appearance, his adventures seem utterly literary. Hence, the article shows that considering Fouke Fitz-Warin as an outlaw is a question not only of rights and politics but also one of aesthetics and literature.
\end{abstract}

Résumé : L'article traite de Fouke Fitz-Warin, modèle du hors-la-loi souvent associé à Robin des Bois. Il s'interroge sur ce qu'est la loi et sur la limite de sa légitimité ainsi que sur la façon dont un hors-la-loi peut paradoxalement incarner le droit et le juste. Ce renversement n'est possible, littérairement du moins, que parce que le personnage n'est pas un simple baron révolté, mais qu'il accède au rang de héros mythique, même si, en apparence, ses aventures peuvent se lire comme purement littéraires. Ainsi, l'article montre que considérer ou non Fouke Fitz-Warin comme un hors-la-loi est une question de droit et de politique, mais également d'esthétique et de littérature.

Le personnage de Fouke Fitz-Warin, tel qu'on le découvre dans l'histoire en prose datée des années 1325-1340', est l'archétype même du hors-la-loi, trickster à ses heures, dans l'imaginaire d'un lectorat qui l'a souvent associé au célèbre Robin des Bois. Comme Robin, il est de ces hors-la-loi, sympathiques redresseurs de torts, qui ne s'opposent à l'ordre établi que pour autant qu'il est inique. C'est là, une grande spécificité du personnage et elle devrait, en elle-même, conduire à nuancer sa classification parmi les hors-la-loi. De fait, au cours de cette analyse, je serai amenée à interroger ce qu'est la loi et quelle est la limite de sa légitimité, et, parallèlement, comment un hors-la-loi peut finalement incarner le droit et le juste, en d'autres termes, une loi véritable, par rapport à la loi corrompue que défend le souverain d'abord reconnu comme légitime. Néanmoins, il me semble que si une telle interrogation, voire un tel renversement du droit et du tort, de la loi et du désordre sont possibles, c'est que le personnage auquel on a affaire a l'étoffe, rare, d'un héros et que sans ce passage par la littérature et notamment par une profonde coloration mythique, Fouke Fitz-Warin ne serait qu'un baron révolté parmi d'autres. On se place ici d'un point de vue littéraire et on ne se situe pas au plan historique.

\footnotetext{
${ }^{1}$ Cette histoire est conservée dans un seul manuscrit : Royal 12.C.XII.

${ }^{2}$ Le trickster, le fripon ou trompeur, par excellence est Loki, membre de la famille des Ases dans la mythologie nordique. Il ne cesse de jouer des tours aux autres dieux. Il a différents avatars dans la littérature européennes et tout particulièrement dans la littérature insulaire britannique qui connaît une influence nordique.
}

Cahiers de Recherches Médiévales, 18, 2009 
L'histoire médiévale de Fouke Fitz-Warin ${ }^{3}$ est l'expression de la situation historique des grands vassaux anglais sous le règne de Jean-sans-Terre ${ }^{4}$. Elle serait le récit de la vie d'un baron anglo-normand du XIII ${ }^{\mathrm{e}}$ siècle. Le matériau historique rejoint l'histoire du biographe, comme en témoignent certaines lettres patentes conservées dans la Tour de Londres. Le roi Jean pardonne au hors-la-loi par trois sauf-conduits puis, l'année suivante, rend une partie de ses fiefs à Fitz-Warin. On connaît un «ordre qu'il adressait à ce sujet au vicomte de Shrewsbury: rex, etc., vicecomiti Salopesbirie, etc. Scias quod reddidimus Fulconis filio Gwarini castellum de Wuitintona cum omnibus pertinenciis suis, sicut jus et hereditatem... $»^{5}$. Le mot jus est d'ailleurs remarquable et rappelle que les revendications de Fouke Fitz-Warin ainsi que sa révolte s'expliquent par l'argument du bon droit. Néanmoins, Fouke entre de nouveau en conflit avec Jean puis avec son fils et successeur, Henri III, qui, dans un ordre au vicomte de Leicester, désigne Fouke comme son ennemi : Fulco filius Warini, qui manifestus inimicus noster est, de eo tenuit in bayllia tua, et est de feodo ipsius comitis ${ }^{6}$. Comme dans l'histoire romanesque, Fouke fait différentes tentatives pour renouer avec le pouvoir légitime mais il a été contraint à demeurer hors-la-loi car la loi officielle était indigne d'elle-même et c'est précisément la mise en récit littéraire de l'histoire qui m'intéresse ici.

\section{Être en lutte contre le souverain légitime suffit-il à faire un hors-la-loi?}

L'Histoire de Fouke Fitz-Warin apparaît en bien des points comme un conte à coloration épique. L'Histoire s'appuie sur la petite histoire, nourrie de surnaturel et de motifs topiques folkloriques ${ }^{7}$. Ainsi, Payn, l'ancêtre de Fouke, rencontre un

\footnotetext{
${ }^{3}$ Pour une approche générale, voir M. Keen, The Outlaws of Medieval Legend, Londres, Routledge et Kegan Paul, 1977 ( $2^{\mathrm{e}}$ éd.). Sur l'histoire de Fouke, voir Louis Brandin, «Nouvelles recherches sur Fouke Fitz Warin», Romania 55, 1929, p. 17-44 ; E. A. Francis, «The Background to Fulk Fitzwarin» dans Studies in Medieval French Presented to Alfred Ewert in Honour of his Seventieth Birthday, Oxford, Clarendon Press, 1961, p. 322-27 ; U. T. Holmes, "The Adventure of Fouke Fitz Warin», dans Medium aevum romanicum: Festschrift für Hans Rheinfelder, Münich, M. Hueber, 1963, p. 179-185 ; M. Zink, «Le rêve avéré. La mort de Cahus et la langueur d'Arthur, du Perlesvaus à Fouke Fitz Waryn », dans Mélanges offerts au Professeur René Fromilhague, Paris, Publications de l'université de Toulouse-le-Mirail, 1984, p. 31-38.

${ }^{4}$ Histoire de Foulques Fitz-Warin, éd. F. Michel, Paris, Silvestre, 1840, p. II. Il existe deux éditions plus récentes : Fouke Fitz Warin: roman du XIV siècle, éd. L. Brandin, Paris, Champion, 1930 (notre édition de référence), et Fouke, le fitz Waryn, éd. E. J. Hathaway et alii, Oxford, B. Blakwell, "Anglo-Norman Texts », 1975. Voir aussi la traduction de G.S. Burgess dans Two Medieval Outlaws. Eustace the Monk and Fouke Fitz Waryn, Cambridge, Brewer, 1997.

${ }^{5}$ Éd. Michel, introduction p. VIII.

${ }^{6}$ Ibid., p. XVI.

${ }^{7}$ Cette alliance entre Histoire et littérature dans les romans anglo-normands est soulignée par Susan Crane dans Insular Romance: Politics, Faith, and Culture in Anglo-Norman and Middle English Literature, Berkeley, Los Angeles and London, 1986. Et elle précise dès la première page de son introduction : «To acknowledge a poem's engagement in the world is
} 
démon issu du fameux géant Géomagog, esprit du diable entré dans le corps du géant $^{8}$, et Payn hérite indirectement du trésor conservé par les géants : c'est la légende de la Blanche Lande. L'intrigue très dense propose exils, voyages en mer, trahisons, travestissements, guet-apens, île déserte, roi sarrasin, nef magique, amours déçus ou autre jeune fille enlevée par un dragon... Néanmoins, cette caractéristique ne peut masquer la problématique profonde du texte : elle présente un puissant baron en lutte presque perpétuelle contre son souverain légitime ${ }^{9}$, en particulier parce qu'il souhaite récupérer la terre de ses ancêtres. Mais le fait d'être en lutte avec un souverain légitime suffit-il à faire d'un homme un hors-la-loi, alors même que, dès son entrée dans le monde adulte, il se présente comme un serviteur du droit? En effet, Fouke, le fitz Warin de Meés, est confié à Joce par ses parents, mais quand le jeune homme a dix-huit ans, Joce se fait attaquer. Fouke se porte alors à son secours et réduit les ennemis conduits par sire Water de Lacy ${ }^{10}$. C'est la première légitimation du personnage, alors qu'il met son bras au service de la juste cause et sort vainqueur. Qui plus est, la féodalité anglo-normande se distingue sensiblement de celle du continent, car la question de la possession de la terre y paraît plus importante : comme le rappelle Susan Crane ${ }^{11}$, l'état de baron dépend davantage de la possession de terres qu'aux qualités guerrières. D'ailleurs beaucoup de barons ne se battaient pas, alors même que nombre de chevaliers ne parvenaient pas à se rendre maîtres d'un territoire. Cet intérêt pour la terre s'explique notamment par la source de richesse agricole qu'elle représente.

La difficulté à qualifier Fouke de hors-la-loi tient à ce que le souverain, quoique légitime, n'est pas toujours un représentant du droit et de la justice. Il semble donc que face à un roi inique, Fouke n'ait d'autre choix que de s'écarter de la loi. Cela dit, la peinture de la figure royale passe par plusieurs étapes. Pour comprendre que Fouke soit considéré comme un hors-la-loi, il faut d'abord dépeindre un roi juste et bon, à l'image des premiers rois anglo-normands dont le charisme avait assuré la paix dans l'aristocratie insulaire. Ainsi quand Fouke le Brun, le fils de Fouke, et les siens sont mis à mal par leurs ennemis, le héros, blessé, va s'en plaindre au roy Henry II, qui le prend sous sa protection avec sa mère et son épouse, alors que son père est mort ${ }^{12}$. Mais «le roy Henri maunda une letre a sire Water de Lacy e comanda sur vie e menbre qu'il ly delyverast Joce de Dynan, son chevaler, e ces chevalers qu'il tient a tort en sa prisoun, e, si yl ne le fet, yl les vendra quere meymes, e fra tiele justice qe tote Engletere en parlera $\rangle^{13}$. Ici, le roi

not to refuse its validity as poetic object. [...] The insular romances deal with the historical world just as surely as they reflect on and liberate themselves form the world ». Le terme de « folklore » est précisément celui qu'emploie E.J. Hathaway pour qualifier les aventures de la seconde partie du texte, op. cit., p. xxxiii. Louis Brandin emploie même le qualificatif de «populaire »: «comme dans les romans d'aventures, des légendes remontant à l'antiquité classique, des contes et anecdotes populaires », op. cit., p. iv.

${ }^{8}$ Éd. Brandin, p. 3.

${ }^{9}$ Éd. Michel, p. I-II.

${ }^{10}$ Éd. Brandin, p. 12.

${ }^{11}$ Op. cit.

${ }^{12}$ Éd. Brandin, p. 27.

${ }^{13}$ Ibid., p. 28, 1. 1-6. 
fait figure de garant du droit et de défenseur de ceux qui subissent l'injustice. C'est lui qui prononce le droit et le tort, comme en témoigne l'emploi du terme «tort» dans le passage cité. D'ailleurs, la légitimité du souverain n'est pas discutée puisque immédiatement, Water obtempère, puis Joce est bien reçu par le roi à Gloucestre. La représentation positive du roi perdure pendant un long moment et correspond à ce que furent vraisemblablement les premiers rois insulaires qui se mêlaient davantage de rendre la justice que leurs homologues continentaux, en sorte que les différends juridiques se réglaient plus souvent à la cour que par la force. Ainsi, "quant sire Joce vint a Gloucestre, le roy le reçust mout léement, et ly promist ley et resoun $»^{14}$. L'auteur insiste sur les termes de «loi» et de «loyauté». Fouke se bat contre Yervard, sur l'ordre du roi, pendant quatre ans, selon les règles des liens vassaliques. À l'issue de cette première période, le roi conclut une alliance avec Yervard, au terme de laquelle ce dernier garde Blanche Ville et la donne ensuite à Rogier de Powis : «si avéz oy coment sire Fouke le fitz Warin de Meez est deherytee de Blanche-Ville e Maylour $\rangle^{15}$. Mais, à ce moment de la narration, il n'y a pas grande contestation. La décision du roi souligne surtout combien le droit du puissant, du seigneur, est différent de celui du vassal. C'est comme si coexistaient deux lois, celle des forts et celle des plus faibles, le système fonctionnant malgré ce déséquilibre, voire s'appuyant sur lui, puisque la féodalité est pyramidale: un puissant, au sommet, s'appuie sur de moins puissants et établit avec eux un réseau de services et de devoirs plus ou moins librement consentis.

Toutefois, à la mort de Richard, présenté comme un roi juste, Johan (Jean sans Terre) règne et l'histoire de Fouke bascule ${ }^{16}$. Morys, descendant de Powis, réclame la Blanche Ville jadis donnée à son ancêtre par Henri. Il pourrait ne s'agir que d'un conflit d'intérêts, puisque le souverain légitime avait effectivement redistribué à Powis le territoire initialement conquis par les ancêtres de Fouke. Chaque personne revendiquant la terre y aurait un droit légitime, mais en fonction de souverains différents. Toutefois la situation se complique surtout en raison des motivations de Johan. Il 'entend avant tout se venger d'une querelle enfantine et superficielle qui a éclaté entre lui, le fils du roi, et Fouke le Brun, alors qu'enfants, ils jouaient aux échecs. Johan n'a pas pardonné à Fouke ${ }^{17}$. Ensuite, Richard Cœur de Lion règne, à la mort de son père, et Fouke le Brun meurt. Fouke, son fils, reçoit son héritage. Du temps du roi Richard, le roi rend à ses descendants les terres qu'avaient perdu Fouke le Brun. À la mort de Richard, c'est Johan (Jean sans Terre) qui règne. Morys, descendant de Powis, réclame la Blanche Ville, jadis donnée à son ancêtre par Henri. Un chevalier a tout entendu et rapporte à Fouke que «le roy confermereit par sa chartre a syre Morys les terres a queux yl avoit dreit $»^{18}$. Bien plus tard, au moment où il agit injustement,

le roy savoit bien qe sire Fouke avoit dreit a Blauncheville, e se remenbra de le coupe qe Fouke ly avoit eynz donee, e se pensa qu'il se vengereit par yleqe, e granta

\footnotetext{
${ }^{14}$ Ibid., p. 28, 1. 12-14.

${ }^{15}$ Ibid., p. 29, 1. 22-24.

${ }^{16} \mathrm{~L}$ 'auteur suit en cela la réputation historique des deux rois.

${ }^{17}$ Éd. Brandin., p. 30.

${ }^{18}$ Ibid., p. 32, 1. 11-13.
} 
qe, quanqe Morys voleyt fere escrivre, yl le enselereyt, e, a ce fere, Morys ly promist cent livrez d'argent ${ }^{19}$.

C'est le moment pour Fouke de la rupture du lien féodal avec le roi et il le manifeste dans ses actes, puisqu'il met à mal des hommes du roi, lancés à sa poursuite. Il s'ensuit que «le roy jura grant serement qu'il se vengereit de eux e de tote lur lignage $\gg^{20}$. Fouke se rend auprès de sa tante puis apprend la mort de sa mère. Il s'en prend à Morys et le blesse. Ce dernier s'en plaint au roi : «le roy devynt si corocé qe a merveyle; e ordina cent chevalers ou lur meynie d'aler par tot Engletere, d'enquere e prendre Fouke e ly rendre al roy vyf ou mort ${ }^{21}$. C'en est fait, Fouke est à présent un véritable hors-la-loi, pourchassé, dont la tête est mise à prix! C'est pourquoi il n'est plus question de légitimité. Un souverain ne saurait faire passer ses rancœurs et ses états d'âme avant le droit. On ne donne pas ici une image positive du souverain puisqu'il ne sait faire passer les intérêts communs avant ses sentiments personnels. Il apparaît plutôt d'un caractère faible, en proie aux anciennes rancunes. Un roi faible justifie-t-il pour autant la rébellion?

C'est, dans une certaine mesure, ce qu'affirme le texte, notamment par l'emploi d'un lexique juridique, en faveur de Fouke. L'histoire de Fouke entretient un rapport essentiel avec les récits consacrés aux barons révoltés. Quand Fouke et son lignage revendiquent leur héritage, c'est leur parole qui s'oppose à celle des descendants de Rogier de Powis. «Fouke e ces quatre freres vindrent devant le roy e prierent qu'il puissent aver la commune ley et les terres a queux yl aveyent droit e resoun, come le heritage Fouke $»^{22}$. Sire Morys s'y oppose, en rétorquant : «Si vus dites qe vus avéz dreit a Blauncheville, vus y mentez » ${ }^{23}$. Un roi juste aurait dû savoir trancher et rendre une exacte justice. Seulement, Johan se préoccupe peu du bon droit et se laisse emporter par un différend futile. C'est finalement le roi qui cause la rupture du lien féodal, comme l'énonce très clairement Fouke, au discours direct, ce qui confère d'autant plus de vigueur et d'autorité à son propos :

Sire roy, vous estes mon lige seignour e a vus fu je lié par fealté, tant come je fu en vostre service e tan come je tienk terres de vous; e vous me dusséz meyntenir en resoun e vus me fayléz de resoun et commun ley, e unqe ne fust bon rey qe deneya a ces frankes tenauntz ley en sa court; pur quoi je vus renk vos homages ${ }^{24}$.

Il est significatif de constater que le lexique du droit et de la féodalité ne sont présents que dans les propos du vassal injustement traité. On remarque «ligeseignour », «fealté », « servise», « homages »... Fouke rappelle les deux éléments que doit faire valoir un souverain juste : « resoun» et « ley». Or « resoun » signifie tout à la fois le «droit», la « justice» et « la parole donnée », « la promesse», ainsi que «le raisonnement», «la pensée» ou «la chose raisonnable». Si «ley», «la

${ }^{19}$ Ibid., p. 32, 1. 3-8.

${ }^{20}$ Ibid., p. 33, 1. 27-9.

${ }^{21}$ Ibid., p. 35, 1. 13-6.

${ }^{22}$ Ibid., p. 32, 1. 13-6.

${ }^{23}$ Ibid., p. 32, 1. 22-3.

${ }^{24}$ Ibid., p. 32, 1. 29-p. 33, 1. 5. 
loi », et «resoun » vont de pair, c'est que la justice s'appuie, non seulement, sur un ensemble de conventions sociales et hiérarchiques féodales, mais, également, sur une pensée juste et une parole exprimant la juste mesure, doublée d'un engagement personnel à la respecter. La justice est ainsi question de parole donnée et de modération. Ici, Johan s'appuie précisément sur la parole donnée pour faire régner l'injustice car il oublie le second impératif: la juste mesure et l'engagement personnel.

De surcroit, dans la suite de ses aventures, Fouke apparaît toujours comme le représentant du droit et de la justice ${ }^{25}$. Fouke tue Rogier et conclut un accord avec le prince Lewys qu'avait épousé la fille du roy Henri. Le prince lui «comaunda la mestrie de tote sa terre $»^{26}$, afin qu'il la défende contre Guenonwyn et détruise le territoire de ce dernier, mais Fouke « fust sages e bien avysee e savoyt bien qe le tort fust al prince $\aleph^{27}$, si bien qu'il s'en ouvre à lui et parvient à lui faire accepter la paix avec Guenonwyn. Ici, Fouke incarne parfaitement la lucidité, le discernement et le choix du droit, y compris contre ses propres amis. Plus tard, le roi de France lui promet de le doter d'un beau territoire s'il reste auprès de lui, alors même que le roi Jean a appris que Fouke était auprès de lui et lui a demandé de le chasser. Mais Fouke répond qu'il ne saurait avoir autorité sur un territoire qui ne lui revient ni par héritage ni par droit. Tout en étant hors-la-loi, Fouke ne renie pas les usages courants, y compris au plan juridique. Fouke a toujours une conduite modérée et sait s'en prendre à ses seuls ennemis : "Fouke ne nul dé suens, de tot le tens qu'il fust exilee, unqe ne voleint damage fere a nully si noun al roy e a ces chevalers $\rangle^{28}$; «mes grant damage firent a le roy par tot e a nul autre si noun a ceux qe furent overtement lur enymys $»^{29}$. Paradoxalement, c'est lui, le hors-la-loi, qui fait le mieux respecter le droit quand le souverain se positionne du côté du tort et de la violence faite aux faibles. La déloyauté royale tente de s'auto-légitimer ici en ayant recours à l'opposition implicite du juste et de la force: celui qui est dans son droit n'a pas besoin d'avoir recours à la force. Par conséquent, qui a recours à la force n'est pas le tenant d'une juste cause. Fouke épouse Mahaut et la sauve ainsi d'un mariage non consenti.

\footnotetext{
${ }^{25}$ «Fulk's protest is just, and in consequence his outlaw life, rather than repudiating law, opposes to the breakdown of justice under King John a principled forest society. [...] Considerable tension arose in the legal sphere between the barony, who were asked to follow court procedures to determine and defend their holdings, and the king, who held himself to be both lawgiver and above the law. The idealized process of these romances intimates not only that law and custom are on the side of the baronial hero and will fulfill the barony's desires, but also that justice is above even kings and will prevail against royal attempts to subvert it », Susan Crane, op. cit., p. 68-69.

${ }^{26}$ Éd. Brandin., p. 46, 1. 30-p. 47, 1. 2.

${ }^{27}$ Ibid., p. 47, 1. 2-3.

${ }^{28}$ Ibid., p. 37, 1. 7-9.

${ }^{29}$ Ibid., p. 41, 1. 23-5. Voir également l'assertion selon laquelle, «Fouke tot cel an entier demora costeant par Engleterre, e a nul home ne voleit fere mal, si noun al roy Johan » (ibid., p. $59,1.23-26)$.
} 
Le roy Johan fust home santz conscience, mavois, contrarious e hay de tote bone gent e lecherous, e, s'yl poeit oyr de nulle bele dame ou damoisele, femme ou fyle de counte ou de baron, e d'autre, yl la voleyt a sa volenté aver, ou par promesse ou par don engyner, ou par force ravyr ${ }^{30}$.

On retrouve ici la mauvaise réputation historique ${ }^{31}$ du roi qui s'explique peut-être par l'image de traitre que véhiculent les moines chroniqueurs reprochant au monarque son refus de prendre part à la Quatrième croisade. Le portrait royal est l'antithèse de celui d'un représentant du droit, comme dans l'épisode au cours duquel Fouke se déguise en charbonnier, car il sait que le roi part chasser, et promet à celui-ci, qui l'interroge, de le conduire jusqu'au gibier, alors qu'il s'agit d'un guet-apens. C'en est fini pour le roi qui demande grâce. Mais il trahit rapidement son serment qu'il estime avoir prononcé sous la contrainte et par trahison Or ce motif fait écho à certains agissements du roi historique puisque Jean, lors de la révolte de nombre de ses barons ${ }^{32}$, a signé la Magna Carta, à Runnymede, près de Londres, le 15 juin 1215, un document qui limitait les pouvoirs royaux et l'une des bases de la démocratie britannique ${ }^{33}$, mais a repris sa parole, avec l'approbation du Pape, en prétendant avoir signé sous la contrainte. C'est avant tout l'anti-modèle moral que met en lumière le narrateur de l'histoire. Une fois encore, Johan se positionne du côté de la force tyrannique, non du droit et de la justice. On assiste à une véritable inversion des valeurs. Fouke peut-il encore être qualifié de hors-la-loi ? Il l'est pour autant que le souverain demeure le référent de toute conduite et que tout comportement qui s'écarte de ses vues est hors-la-loi ${ }^{34}$.

\footnotetext{
${ }^{30}$ Ibid., p. 49, 1. 7-12.

${ }^{31}$ Voir Sidney Painter, The Reign of King John, Baltimore, John Hopkins Press, 1949.

${ }^{32}$ De manière générale, au cours de son règne, Jean s'est attiré l'inimité des barons en leur imposant des taxes plus lourdes que n'impliquaient les règles féodales traditionnelles.

${ }^{33}$ «Magna Carta (1215) was the greatest single expression of this peculiarity of AngloNorman life. Magna Carta sought not to reestablish freer relations between king and barons, but to incorporate the king into his own legal system, to restrain him, too, within the fine new net of law he had cast around his barons. [...] Such was the milieu, tenaciously legalistic yet adaptable and practical, in which the romances of English heroes were written », Susan Crane, op. cit., p. 21.

${ }^{34}$ Il faut nuancer cette assertion dans la mesure où, depuis la Magna Carta, les pouvoirs du roi anglais sont affaiblis par rapport à ceux de son homologue français, au point que «Saint Louis, lors de la Mise d'Amiens en 1264, ne saurait comprendre pourquoi on sollicite son arbitrage dans la querelle opposant les barons au souverain britannique: pour lui, les prérogatives royales ne peuvent faire l'objet d'aucune discussion. Nul arbitrage n'est possible, le roi a forcément raison. Le roi ne peut partager le pouvoir. La conception monarchique française aboutit à l'absolutisme, celle de l'Angleterre au parlementarisme ", Florence Bourgne, Leo Carruthers et Arlette Sancery (dir.), Un Espace colonial et ses avatars. Naissance d'identités nationales : Angleterre, France, Irlande ( $V^{e}-X V^{e}$ siècles), Paris, Presses de l'Université Paris-Sorbonne (Cultures et Civilisations médiévales, 42), 2008, p. 11. Jean perd même son propre héritage, la Normandie en 1204. La France et l'Angleterre divergent alors au plan politique malgré leur culture commune (voir Andrew Baume, « the context and consequences for England of the loss of Normandy in $1204 »$, ibid., p. 91-102). Susan Crane
} 
D'ailleurs, Fouke joue de son emploi, de son rôle de hors-la-loi. Un malhonnête à la tête d'une bande de larrons se fait appeler Fouke et vole et pille autour de lui. Le véritable Fouke ne supporte pas une telle supercherie, il trouve le traître usurpateur et lui coupe la tête. Ce faisant, Fouke fait preuve d'une volonté farouche de se comporter non selon la loi, mais selon une loi supérieure qu'est son sens moral : il ne s'agit plus de rendre des comptes au roi, mais à Dieu. Néanmoins, Fouke joue de sa fausse réputation et il laisse penser aux gens de la Blanche Ville qu'il est mort, lors d'un vol qu'il aurait voulu commettre. Le personnage joue ici de son «emploi », au sens théâtral, et il devient du même coup maître de la fiction, au plan littéraire. Encore une fois, Fouke ne fait que mettre en lumière l'absence de discernement du roi qui pourtant devrait être au fondement de son sens de la justice : sa fameuse «resoun». Par contraste, Fouke sait agir avec discernement, même quand il s'agit de mettre en cause ses amis, y compris le prince Lewys, son allié, dont il sait qu'il a tort dans son conflit avec Guenonwyn. Ainsi la figure du hors-laloi sert aussi de prétexte, de moyen détourné pour questionner la figure royale et sa légitimité. Il semble bien que l'histoire de Fouke exprime la mauvaise réputation traditionnelle de Jean-sans-Terre, puisqu'on rencontre dans l'œuvre d'autres figures royales qui incarnent parfaitement l'idéal du souverain légitime. C'est le cas du roi de France, Philippe, qui reconnaît instinctivement que Fouke est un homme de valeur et qui va jusqu'à lui proposer de lui donner un territoire.

Mais l'histoire de Fouke est également l'occasion d'une réflexion sur le sens du territoire puisque, quand Philippe lui propose un fief, Fouke répond: «certes, sire, [...] yl n'est pas digne de receyvre terres de autruy doun, que les suens de dreit heritage ne puet tenir a reson $»^{35}$. Il est intéressant de remarquer que si les qualités nécessaires à un souverain juste font défaut à Johan, elles sont le propre du hors-laloi Fouke. Il les exprime d'ailleurs en des termes très proches de ceux qui dénonçaient l'imposture royale. On retrouve la question morale de la « dign[ité]» et de ce qui est «dreit» et, surtout, on répète le mot «reson». Ce sont deux conceptions de la justice qui s'opposent : l'une, celle de Johan, s'appuie sur sa seule qualité de roi, l'autre, celle de Fouke, sur une vertu morale. C'est encore à un lexique moral plus que juridique que Fouke fait appel pour exprimer son choix de devenir hors-la-loi : «Fouke jura grant serement qe pur pour de moryr ne lerreit qu'il ne se vengeroit de le roy, q'a force e a tort ly ad desherytee, e qu'il ne chalengereit hautement ces dreytures e son herytage $»^{36}$. Du côté du roi sont « force» et «tort», alors que de celui de Fouke sont «dreytures» et « grant serement». De nouveau, avec le «serement», on renoue avec l'alliance entre engagement moral et

explique : «the romances of English heroes acknowledge the dominance of national ideology by recognizing the right and power of kings, placing high value on communal stability, and representing the legal system as a legitimate source of redress for the barony. But at the same time, these romances reinterpret nationalism to the advantage of the newly constrained barony. The desires of the noble hero subsume the desires of his community [...]. Through this pattern the romances of English heroes consistently subvert the political principle that royal and national interests must come before those of landed barons » (op. cit., p. 218).

${ }^{35}$ Éd. Brandin, p. 57, 1. 25-7.

${ }^{36}$ Ibid., p. 68, 1. 6-10. 
parole donnée. Ce qui est considéré comme juste semble être l'adéquation entre la parole proférée et l'action.

Néanmoins, dès lors que la légitimité du garant de l'ordre est remise en cause, les règles de la justice sont sans cesse bafouées. Même Fouke qui semble pourtant être le modèle du droit, se livre à de petits arrangements moraux que le narrateur de l'histoire justifie aisément, par la bassesse du personnage de Johan, qui n'est pas dépeint comme un homme qui mériterait une grande considération morale, et par la tonalité légère de certains épisodes. Il en est ainsi quand Fouke se déguise en charbonnier, alors qu'il sait que le roi qui l'interroge va chasser, et qu'il lui promet de le conduire jusqu'au gibier, tout en ayant l'intention de lui tendre un guetapens. Là, la ruse l'emporte sur la justice pure. Mais peut-on avoir recours à la ruse pour faire valoir son bon droit? Est-ce légitime? Il ne semble pas que cela soit pleinement recevable dans la morale médiévale. On réserve d'ordinaire plutôt la tactique du guet-apens aux anti-héros. Mais, nous l'avons dit, on assiste à un renversement total des valeurs puisque lors de ce guet-apens, le roi apeuré n'hésite pas à prononcer un faux serment et à tromper Fouke pour qui la parole donnée est sacrée :

le roy li cria mercy, e ly pria pur amour Dieu la vie e yl ly rendreyt enterement tou son heritage et quanqu'il aveit tolet de ly et de tous les suens, e ly grantereit amour e pees pur tous jours, e, a ce, ly freit en totes choses tiele seureté com yl meysmes voleit devyser. Fouke ly granta bien tote sa demande a tieles qu'il ly donast, veantz ces chevalers, la foy de tenyr cest covenant. Le roy ly plevy sa fey q'il ly tendroit covenant $(\ldots)^{37}$.

On constate là encore combien le roi pèche contre le sens de la justice. Comme pour amoindrir la valeur performative de la parole royale, le narrateur la présente d'ailleurs au discours indirect, au rebours de celle de Fouke précédemment. Elle perd ainsi en intensité. Le roi a recours à des termes juridiques : "grantereit» ou « covenant». Il fait fi de sa propre parole, la «fey» qu'il «plevy» : on constate chez lui une inadéquation entre la parole et l'action. Mais peut-être plus grave encore, le roi pèche contre les fondements même de son propre pouvoir et de son hypothétique justice : celle d'un grand seigneur par rapport à son baron ou à son vassal, puisqu'il prête un faux serment "veantz ces chevalers ». Il leur ment indirectement et les rend témoins et garants d'un faux discours, ce qui est gravissime dans la pensée médiévale. Plus funeste encore, le roi est injuste et traître sous le regard de Dieu à Qui il en appelle pour susciter la pitié de Fouke. On a affaire à un roi sacrilège. Peutil encore rendre la justice? Est-on hors-la-loi quand on s'oppose à un souverain sacrilège?

Ce n'est qu'acculé que le roi retrouve le sens de la justice en même temps que le sentiment du sacré. Quand Johan sent qu'il ne peut plus faire face à Fouke, il «s'en ala a Westmostier, e fist assembler countes, barouns e la cleregie, e lur dit apertement qu'il avoit de gree graunté sa pees a Fouke le fitz Warin ${ }^{38}$ et aux siens

${ }^{37}$ Ibid., p. 69, 1. 25-p. 70, 1. 3.

${ }^{38}$ Ibid., p. 81, 1. 3-6. 
« e lur graunta entierement tot lur heritage $»^{39}$. Fouke capture le roi qui chassait le sanglier et ce dernier promet, à cette occasion, de lui rendre son héritage. Il le fait finalement et il s'ensuit une nouvelle allégeance, logique et juste, au roi à Londres ${ }^{40}$, après plus de deux ans de vie de hors-la-loi. Le juste est rétabli dans ses droits, mais la société n'est pas bouleversée. Fouke rejoint ensuite le comte Rondulf de Cestre et l'aide à défendre ses droits en Irlande. Il ne semble pas varier du début à la fin de l'œuvre. Il offre une fois encore sa confiance au roi et lui fait une nouvelle allégeance. Le portrait moral du hors-la-loi est d'ailleurs encore plus exigeant :

Fouke se purpensa qu'il avoit grantment meserré countre Dieu, come en occisioun des gentz e autres grauntz meffetz, e, en remissioun de ces peschiés, founda une priorie en le honour de Nostre-Dame seinte Marie, de le ordre de Grantmont, pres de Alberburs, en le boschage sur la rivere de Sauverne, e si est appelee la NoveleAbbeye ${ }^{41}$.

Jusqu'au bout, Fouke est l'anti-Johan. Même si le lecteur sait que Fouke n'a jamais commis de crime contre un innocent, mais s'est seulement défendu de ses ennemis, le héros sent la faute morale que commet tout homicide. Il ne se contente pas d'incarner le Juste devant les hommes, il s'emploie également à rendre des comptes à Dieu. Contrairement au roi, Fouke associe au juste le sacré.

\section{La coloration mythique}

Ce qui permet à Fouke d'être à la fois héros et hors-la-loi, dans une pensée médiévale où la loi féodale domine et où il ne fait pas bon s'opposer au souverain légitime, est sa dimension mythique. Son caractère héroïque est reconnaissable à différents traits. Ses ancêtres ont la trempe de héros civilisateurs. Payn Peverel lutte avec les puissances de l'autre monde sous la forme d'un démon issu du géant Géomagog; on va même jusqu'à dire que c'est l'esprit du diable entré dans le corps de Géomagog ${ }^{42}$. Or, dans la pensée et la littérature médiévales, les êtres capables de tels exploits appartiennent souvent un peu à cet autre monde. Arthur, par exemple, vainqueur de géants, est un souverain mythique. Ce lien avec le monde gigantal est un gage d'héroïsme. Par sa victoire contre le démon, Payn hérite indirectement du trésor conservé par les géants et fonde la légende de la Blanche Lande. La possession même de la Blanche Ville se présente au début de l'œuvre comme mythique, puisqu'elle procède de la victoire sur un géant et de la discussion avec son esprit. À ancêtre mythique, descendant héroïque !

Fouke confirmera cette ascendance mythique de façon de plus en plus explicite. Dans un premier temps, on peut lire ses aventures au plan réaliste et voir dans le fait qu'il habite les forêts (celles de Wundesoure, de Bradene, de Kent ou la Nouvele-Forest) la simple conséquence de sa condition de hors-la-loi, condamné à

${ }^{39}$ Ibid., p. 81, 1. 7-8.

${ }^{40}$ Ibid., p. 81.

${ }^{41}$ Ibid., p. 83, 1. 1-7.

${ }^{42}$ Ibid., p. $3-7$. 
l'errance et à la discrétion ${ }^{43}$. En effet, au Moyen Âge, la forêt n'est pas un lieu qui a bonne réputation. Mais il faut penser aussi à la charge mythique de la forêt médiévale qui abrite monstres et merveilles ou permet de passer dans l'autre monde. Fouke et les siens sont associés à différents espaces naturels et/ou sacrés. Mahaud, l'épouse de Fouke, accouche dans la nature ou dans les églises... Le héros apparaît sous la protection du monde naturel et, à travers lui, de Dieu qui préside à la nature. Le Juste est toujours protégé par le Seigneur qui sonde les cœurs et les reins, c'est pourquoi il finit par avoir le dessus sur le roi Johan et ne perd pas espoir. Fouke incarne parfaitement la figure du Juste, notamment par l'outrance héroïque dans les qualités: "Fouke avoit tele grace qu'il ne vynt unqe en nul lyu ou hardiesse, chevalerie, prouesse ou bountee fust, qu'il ne fust tenuz le meylour e santz pier $\rangle^{44}$. La façon dont Fouke, au terme de sa lutte contre Johan, reprend le pouvoir utilise des scénarii mythiques. Le roi est capturé par Fouke dans la Nouvele-Forest alors qu'il est à la chasse au sanglier, et ce n'est pas un hasard. Les chasses aux sangliers sont fréquemment l'occasion d'événements surnaturels ou, du moins, hors du commun dans la littérature médiévale.

Mais la suite des aventures de Fouke, à partir du moment où il s'engage en mer, lors de son exil, est empreinte d'une nouvelle coloration. Ce n'est plus seulement l'histoire d'un baron révolté, c'est l'expédition d'un héros civilisateur et redresseur de torts, seul capable d'affronter des monstres et de faire preuve de noble chevalerie. Fouke va d'île en île. Il se rend notamment sur Eschavye où il existe des serpents "volantz come oysels " ${ }^{45}$, avec lesquels il se bat; ce sont toutes les créatures venimeuses chassées d'Irlande par saint Patrick qui sont enfermées dans l'île. Fouke s'achemine vers l'image traditionnelle du héros qui refonde la civilisation contre la barbarie. Celle-ci est par exemple représentée par le dragon qui a enlevé la fille du duc de Cartage, qu'il oblige à nettoyer sa barbe à chaque fois qu'il s'est repu d'êtres humains. Fouke le vainc et libère la jeune fille. Le tort infligé par un représentant du droit inviterait à refonder une société juste ailleurs. Quand Fouke, à la fin de ses aventures, rejoint le comte Rondulf de Cestre et l'aide à défendre ses droits en Irlande, l'un des ennemis est un géant « hidous, neir e orrible, plus long que nul autre de douze piés $\gg{ }^{46}$. Il tue les chevaliers les uns après les autres avec sa hache. Fouke en est vainqueur, en le décapitant. En perpétuant le geste de son ancêtre, il rappelle sa légitimité et sa puissance hors-norme, qui font de lui intrinsèquement un hors-la-loi, c'est-à-dire un être qui n'est pas soumis aux lois communes. Ses aventures se déroulent comme si une collusion existait entre le droit et le mythe. Seul un héros mythique paraît pouvoir être le véritable garant d'un droit qui dépasse la soumission aux autorités politiques... En outre, au plan historique, c'est par l'expression de leur valeur chevaleresque que les barons anglo-normands vont justifier leurs droits déclinant à la fin du XIII ${ }^{\mathrm{e}}$ et au $\mathrm{XIV}^{\mathrm{e}}$ siècles, comme en témoigne le personnage de Fouke. C'est le caractère héroïque de Fouke qui fonde son droit à la désobéissance.

${ }^{43}$ Par exemple, Fouke « vient en la foreste de Kent e lessa ces chevalers en l'espesse de la foreste », ibid., p. 37, 1. 17-8.

${ }^{44}$ Ibid., p. 56, 1. 23-5.

${ }^{45}$ Ibid., p. 63, 1. 9-10.

${ }^{46}$ Ibid., p. 82, 1. 8-9. 
Fouke n'est d'ailleurs pas seulement un tueur de monstres, il est également un héros par la dimension sacrée de son action. Il conduira ceux qui sont dans l'erreur vers le seul vrai Dieu, comme avec le roi de Tunes, le sarrasin Messobryns, en guerre car il veut épouser la fille du duc de Cartage, alors qu'elle le refuse. Fouques lui promet de défendre sa cause à condition qu'il se convertisse au christianisme et se fasse baptiser. Fouke est élu de Dieu et communique de façon privilégiée avec le divin. Ainsi, une nuit, Fouke pense aux méfaits de sa jeunesse et voit une grande clarté. Une voix s'adresse à lui : "vassal, Dieu te ad graunté ta penaunce, que mieux valt ci qe aillours $\gg{ }^{47}$. L'épouse du héros entend la voix et perçoit la clarté. Depuis ce jour, Fouke reste aveugle en pénitence. Or cette cécité n'est pas sans rappeler d'autres handicaps mythiques, comme l'aveuglement de Paul sur le chemin de Damas ou celui de nombreux devins. Loin d'être une infirmité, elle souligne la carrure mythique du personnage.

L'ensemble de ces traits mythiques est souligné par le rappel de la prophétie de Merlin, déjà énoncée au début du texte, par l'esprit démoniaque interrogé par Payn Peverel, à la fin de l'œuvre :

En Bretaigne la Graunde

un lou vendra de la Blaunche Launde;

XII. dentz avera aguz,

sys desouz et sis desus.

Cely avera si fer regard

qu'il enchacera le leopard

hors de la Blaunche Launde :

tant avera force et vertue graunde.

Mes ne le savom qe Merlyn

le dit pur Fouke le fitz Waryn,

quar chescun de vus deit estre ensur

qe en le temps le roy Arthur

la Blanche Launde fust appelee

qe ore est Blaunchevile nomee ${ }^{48}$.

Ce rattachement ultime à la figure de Merlin assure un retentissement surprenant à la rébellion du baron révolté. L'histoire de Fouke se trouve rehaussée par la caution de la meilleure tradition littéraire romanesque.

En raison de cette collusion avec une puissance surnaturelle issue de l'autre monde, le lecteur pourrait faire le rapprochement entre Fouke et Huon de Bordeaux, héros éponyme d'une chanson de geste du XIII ${ }^{\mathrm{e}}$ siècle $^{49}$, injustement dépossédé de son territoire par Charlemagne, dont il a accidentellement tué le fils. Or l'histoire de Huon vient renforcer nos présomptions. Huon reste toujours fidèle et soumis à

${ }^{47}$ Ibid., p. 83, 1. 29-30.

${ }^{48}$ Ibid., p. 84, 1. 10-23. Ce passage se singularise par sa forme versifiée qui témoigne vraisemblablement d'un état texte antérieur à celui qui nous est parvenu, sans doute daté de la fin du XIII ${ }^{\mathrm{e}}$ siècle (selon E.J. Hathaway, op. cit., p. xix-xx).

${ }^{49}$ Huon de Bordeaux, éd. et trad. William W. Kibler et François Suard, Paris, Champion (Champion classiques), 2003. 
Charles, à la différence de Fouke qui s'érige en hors-la-loi et se soustrait à la souveraineté de Johan. Pourtant Huon reçoit un important soutien surnaturel pour la récupération de son fief, dans l'aide d'Aubéron, petit roi de Féerie. Mais, là est précisément ce qui distingue les deux personnages. Huon n'a pas la carrure mythique de Fouke. Huon, lui, ne serait parvenu à rien sans son auxiliaire Aubéron. D'ailleurs, les derniers mots de celui-ci avant de quitter le monde des hommes le prouvent puisqu'ils interdisent à Huon de jamais se quereller avec le roi : «tez sire est, se li dois foid porter» (v. 10765). C'est pourquoi on n'a pas affaire à une véritable problématique juridique dans Huon, concernant le territoire et l'héritage, alors qu'avec Fouke, on pose de manière frontale la question de la légitimité d'un territoire et de la valeur d'un héritage sur le plan de la loi et du droit.

Voir en Fouke Fitz-Warin un hors-la-loi est une question de droit ou de politique, mais également une question esthétique et littéraire. Fouke est un hors-laloi et un héros littéraire à la fois, le récit de son existence revêt un caractère mythique que rend sensible un travail efficace sur les ruptures de tonalités. Fouke Fitz-Warin est une œuvre historique et politique qui ne perd jamais sa cohérence, que le protagoniste soit hors-la-loi ou puissant aventurier. Qui aurait cru, au moment où Fouke se voit spolié de son héritage et s'oppose au roi Johan, le voir affronter un dragon mangeur de jeunes femmes? Rien de prévisible dans le récit, si ce n'est sa ressemblance avec d'autres romans lignagers anglo-normands, comme le montre Maria Dominica Legge. Le texte s'intéresse au destin d'une famille depuis la conquête normande jusqu'au XIII ${ }^{\mathrm{e}}$ siècle. Ce genre de la chronique familiale connaît d'autres représentants: Guillaume d'Angleterre (fin XII ${ }^{\mathrm{e}}$ siècle), Waldef (fin XII siècle), Beuve de Hantone (fin XII ${ }^{\mathrm{e}}$ siècle), Fergus (XIII ${ }^{\mathrm{e}}$ siècle) ou Gui de Warewic (XIII ${ }^{\mathrm{e}}$ siècle). Ces histoires ont différents éléments communs, qui minimisent en apparence l'originalité de l'histoire de Fouke: le héros doit être le fondateur de la famille, il est exilé à l'étranger, il vit des aventures merveilleuses, et doit notamment venir à bout d'un dragon, il se réconcilie à la fin avec le roi et retrouve son héritage, son mariage et sa descendance sont clairement exposées, afin de rendre compte de sa généalogie, il est enterré dans un monastère qu'il a fondés ${ }^{5}$. Comme dans Hereward le Proscrit ou l'Exilé, Eustache le Moine, on retrouve le roman dont le héros est un hors-la-loi, dont les aventures mêlent histoire, légende et mythe et qui accorde une puissance manifeste à la justice ${ }^{51}$.

Toutefois, cela ne doit pas masquer la singularité et la valeur littéraire de l'histoire de Fouke ${ }^{52}$. L'auteur y joue des métamorphoses du personnage, tant à un

\footnotetext{
${ }^{50}$ Maria Dominica Legge, Anglo-Norman Literature and Its Background, Oxford, Clarendon Press, p. 170-175.

${ }^{51}$ Voir à propos de la justice dans les romans anglo-normands, Susan Crane, op. cit., p. 67-73.

${ }^{52}$ «In Fouke, a remarkable historical moment, an exceptional setting, and a heavily romanticized account of the Fitz Warin family's affairs allow the ideal fictional pattern of baronial victory to play itself out in a situation from the insular barony's own history », Susan Crane, op. cit., p. 58.
} 
niveau matériel, par les déguisements qu'il revêt ${ }^{53}$, qu'au plan de l'esthétique, par le genre de personnage que Fouke incarne, à la fois baron révolté et héros civilisateur. L'unité de l'histoire est d'ordre mythique. Le retour du hors-la-loi sous l'autorité royale, la nouvelle allégeance, l'illustre: elle est précédée par une refondation symbolique de la civilisation par le héros, comme si, pour rétablir le droit, il fallait passer par une réinvention du monde perverti par un ordre injuste. Mais plus intéressant encore, en termes de littérature, est que Fouke se rapproche du littérateur, au sens de faiseur de fiction, à différents plans. Fouke invente une fiction pour expliquer ses blessures, lorsqu'il est recueilli à Tunes par le roi Messobryns et sa sœur Isorie : il propose une histoire tout à fait romanesque de trahison et de jalousie amoureuse, en rupture de ton totale avec ce qui précède. Cet exercice du héros met en valeur sa ruse tout comme elle est emblématique de la large palette du narrateur de l'histoire qui sait écrire dans des genres très contrastés. Les différents et abondants déguisements de Fouke sont également autant de métaphores de la fiction. Le personnage est un créateur de personnages, comme peut l'être l'écrivain. Mais si Fouke peut être justifié en tant que hors-la-loi, ce n'est pas en raison de son attrait littéraire, mais seulement grâce à sa nature mythique. Toutefois, de façon générale, le passage d'un personnage exprimant des conflits historiques par la littérature permet que devienne figure centrale la figure marginale du hors-la-loi historique. Le personnage gagne en puissance, se nourrit de l'imaginaire pour suppléer à sa faiblesse historique. De plus, la littérature est bienveillante et accueillante pour le hors-la-loi : il peut y créer ses propres règles, ses propres lois.

Myriam White-Le Goff Université d'Artois (Arras)

«Textes et Cultures » (EA 4028)

ANR Juslittera

${ }^{53}$ Par ce motif du travestissement, il faut rapprocher Fouke du Tristan anglo-normand, exilé comme lui, qui ne peut revoir sa bien-aimée et la cour du roi Marc que sous une apparence trompeuse. 